\title{
A Critical Review: Spinning Methodologies, Properties and Applications of Graphene Fibre ${ }^{\star}$
}

\author{
Yangyang Fan, Yi Li*, Xuqing Liu \\ School of Materials, University of Manchester, Oxford Road, Manchester, M13 9PL, UK
}

\begin{abstract}
Inspired by macroscopic high dimensional graphene materials, graphene fibres now have been fabricated in a range of methods, including drawing, solution spinning, thermal process, electrospinning, etc. Among them, most attractive, large-scale and widely used methodology is solution spinning liquid crystal graphene oxide, including wet spinning and dry-jet wet spinning. Properties of different kinds of graphene fibres were discussed in this review. Both neat graphene fibres and hybrid graphene fibres show remarkable mechanical and electrical properties, actuating properties and flexibility. Multifunctional properties provide graphene fibres potential in functional textiles, flexible and wearable sensors, supercapacitors, electrodes of supercapacitors, energy devices, and actuators etc. It is prospected that properties of graphene fibres will be further optimized, and their application will be more realistic and well developed.
\end{abstract}

Keywords: Graphene Fibres; Spinning; Mechanical Properties; Electrical Properties; Application

\section{Introduction}

\subsection{Introduction to Graphene}

Graphene firstly found in 2014, by A.K. Geim and K.S. Novoselov [1], has been widely seen as a honeycomb structure of two-dimensional (2D) atomic crystals with high crystal quality and macroscopic continuity. Graphene can be seen as a single layer exfoliated from multiple layer graphite, a plat structure rolled out from single-wall carbon nanotubes or fullerene molecule $[2,3]$. The most attractive factor of graphene is its extraordinary properties, with an ideal tensile strength of $130 \mathrm{GPa}$, elastic modulus of $1.0 \mathrm{TPa}$, remarkable flexibility, electrical conductivity of $10^{8} \mathrm{~S} / \mathrm{m}$, thermal conductivity of $5000 \mathrm{Wm}^{-1} \mathrm{~K}^{-1}$, transmittance of almost $97 \%$ and high temperature, chemical resistance [4]. Owing to these desirable properties, graphene was not only not only made into a neat structure, but also hybrid with other material.

${ }^{\star}$ Project supported by EU Horizon 2020 and University of Manchester (AA14512, AA01906).

${ }^{*}$ Corresponding author.

Email address: henry.yili@manchester.ac.uk (Yi Li). 


\subsection{Graphene Based Materials}

\subsubsection{Two-Dimensional (2D) and Three-dimensional (3D) Graphene Materials}

First graphene films were prepared by continuous mechanical exfoliation of small mesas of highly oriented pyrolytic graphite. After this simple but reliable method, FLG films were found up to $10 \mu \mathrm{m}$ in size. However, clearly, unrealistic and unfeasible problem, approach of making single layer graphene should be optimized or substituted. After micromechanical cleavage, several methods were developed and have been widely applied in making experimental graphene, e.g. acid oxidation, chemical vapor deposition etc $[5,6]$. Graphene oxide formed in some cases will then be reduced using thermal or chemical methods [7, 8].

Graphene has demonstrated excellent mechanical, electrical, thermal, and optical properties [4, 6-11]. It is assumed that this will bring graphene great potential in many high technical fields both experimental and practical because assembling 2D graphene nanosheets into 3D graphene structure is the most realistic and efficient approach to utilise its extraordinary properties [9]. Therefore, the most important issue of graphene is to transform and fabricate micro scale graphene into macroscopic structures and to make it functional for practical applications.

The assembly of graphene into macroscopic three-dimensional (3D) structures has been attracting intensive interest [10-12]. Till now, three-dimensional graphene aerogel, hydrogel, foams, sponges [9, 13-17] and two-dimensional graphene film/member/paper/nanosheets [5, 18, 19].

For 3D graphene structure, it is illustrated that 3D graphene achievements have been made in graphene 3D structure on their use in electrode of supercapacitor, supercapacitors, conductors with flexibility and biosensors etc $[13,20]$.

Other improvements in high-performance 2D films, paper, membrane etc. have spurred research efforts on their application in electric double-layer capacitors and pseudo-capacitors stretchable electrodes, biosensor, biomedical applications due to their acceptable mechanical strength, electrical conductivity, signal-to-noise ratio and potentially biocompatible [5, 9, 18, 19].

\subsubsection{One Dimensional (1D) Graphene Fibres}

In contrast, among macro scale graphene material, 1D graphene fibre was believed to emerge high electrical conduction, ultrahigh mechanical strength after stretching, functional possibility, unique flexibility and weavability compared with the other two kinds of graphene macroscopic materials. Hence, graphene fibre has outstanding potential in wearable smart textile, biosensor etc. Provided that structure of graphene fibre is continuous with 2D carbon monocrystals stacked together along fibre axial direction, graphene fibre is also designed with a purpose of overcoming the shortages of conventional carbon fibre PAN, e.g. vast grain boundaries and polycrystalline nature [21].

Nowadays, one-dimensional graphene fibres have already been prepared and confirmed with multiple superior properties compared with conventional large scale material. As a novel material, graphene fibre is fabricated to fill in gaps of one-dimensional graphene material, and to prepare multifunctional fibres with proper qualities in some specific areas.

In this review, we concluded current methodology with principle of fabricating macroscopic multifunctional graphene fibres. Thermal, electrical, mechanical properties of neat graphene fibres from different methodology were compared and discussed. Strategies of doped graphene 
based fibres were also introduced. Finally, the current application of graphene fibres and their potential usage were rendered to lead to a positive prospect in fibre production field.

\section{Graphene Fibres Spinning Methodology}

In contrast to high-dimensional material have already been made such as aerogels and membranes, graphene-based fibres were demonstrated to have remarkable mechanical, electrical properties alone fibre axis direction, and in some conditions can be knottable and weavable [22]. To fabricate a 2D graphene structure, common methods of polymer spinning, e.g. melt spinning, dry spinning, wet spinning, dry jet-wet spinning and electrospinning were taken into account.

Graphene, well-known as an extremely thermal stable crystalline material, has a melting temperature $\mathrm{T}_{m}$ around $4900 \mathrm{~K}$ (about $250 \mathrm{~K}$ higher than that of graphite, probably the highest in nature) [23]. In this respect, it is unrealistic to make neither neat graphene fibres nor graphene based composite fibres using melt spinning approaches.

In dry spinning, spinnability is influenced by both solution rheological properties and mass transfer rates. Solution rheological property is commonly known as one of the key factors to determine spinnability of dry spinning [24]. However, lacking entanglement of 2D graphene leads to a relatively low solution reheological property. It highly restricts graphene fibre fabrication using continuous dry spinning. According to these natures of graphene, self-assemble graphene aqueous together with other methods were taken into consideration next section.

\subsection{Drawing Graphene}

First neat graphene fibres were attempted to by drawing graphene macrosheets [25]. In this novel yet simple method, 2D graphene film prepared early by chemical vapor deposition was self-assembled into a 1D fibre structure. The short fibre was then dried showing a porous and crumpled morphology. However, only van der Waals force existed between graphene sheets after being dried indicating inferior mechanical properties.

As the porous structure giving a potential in electrode material, manganese dioxide was added into graphene substrate. Following electrochemical characterisation confirmed that with incorporating manganese dioxide, graphene based fibre showed a tremendous improvement in electrochemical properties. Ever since, many other approaches of preparing macro scaled graphene were developed.

\subsection{Solution Spinning}

\subsubsection{Liquid Crystal Graphene Oxide}

Liquid crystal graphene (LC GO) is the essence of wet spinning. LC GO phase was firstly observed by Natnael et al when graphene was dissolved in chlorosulphonic acid. The superacid contributed to exfoliation and protonation of graphene, as a consequence of which induced repulsion between graphene layers. A spontaneous Liquid crystalline graphene phases appeared at high concentration dispersion $\left(20 \mathrm{mg} \mathrm{mL}^{-1}\right.$ to $\left.30 \mathrm{mg} \mathrm{mL}^{-1}\right)$. After that, more LC GO were found and investigated in different other solutions [26]. Gao C.'s group firstly reported that soluble, 

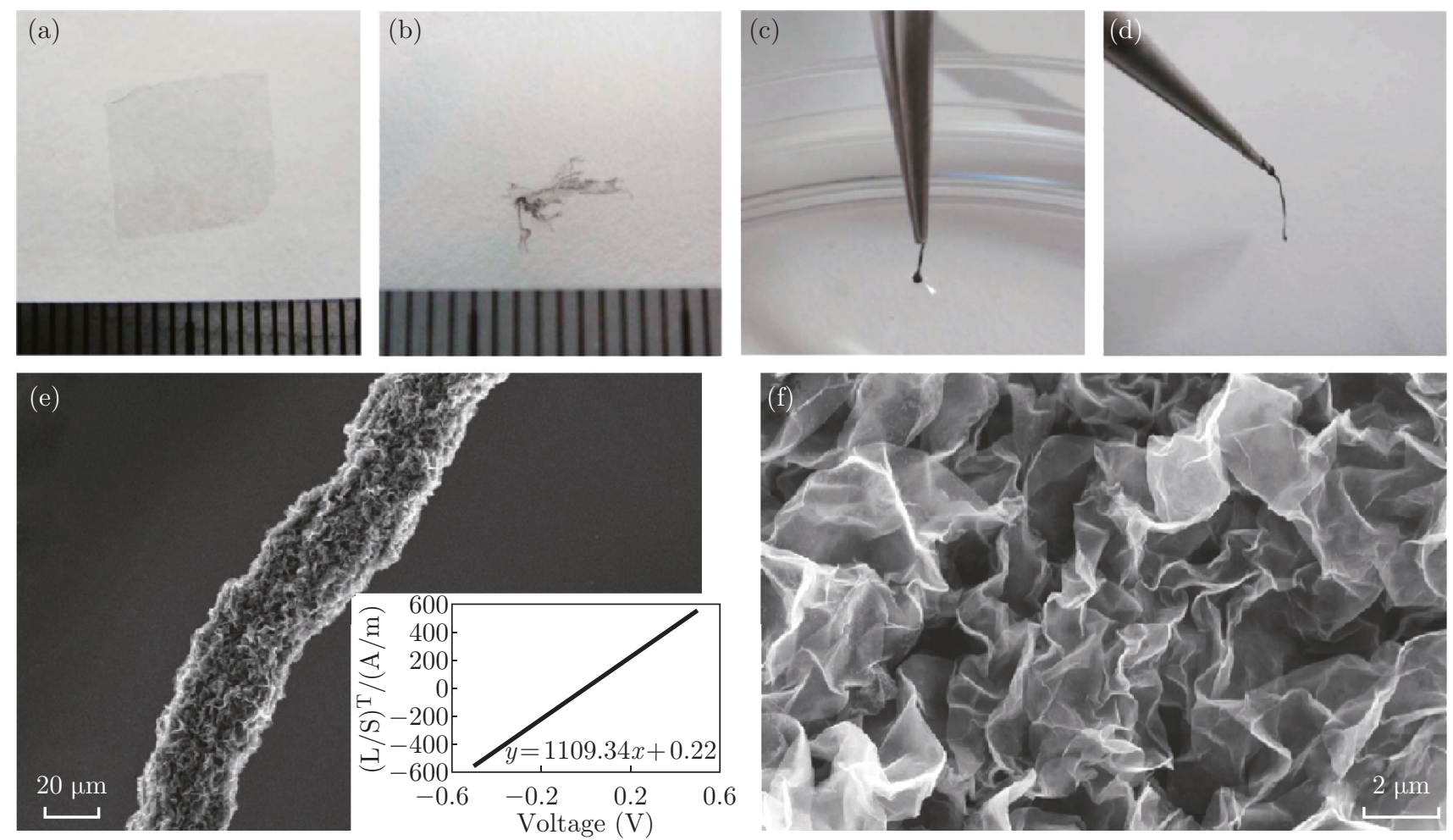

Fig. 1: (a) Flat graphene film in deionized water. (b) Twisted and wrapped graphene film in ethanol. (c) Graphene fibre formed by drawing out of ethanol. (d) Graphene fibres with twisted structure. (e) SEM image of single graphene fibre with rough surface. (f) SEM images of single graphene fibre, showing porous structure [25]. Copyright 2011, American Chemical Society

chemically oxidized graphene or graphene oxide sheets can form chiral liquid crystalline (Fig. 2) [27]. One of the most important applications of LCs is to make macro scale fibres using wet spinning. Gordon W.' group then investigated the quantitative relation between GO nanosheets size and GO concentration, liquid crystallinity, spinnability etc [28-31].

According to these researches, two key factors are demonstrated to form liquid crystalline graphene: aspect ratio and dispersibility or solubility. Aspect ratio was known as width/thickness
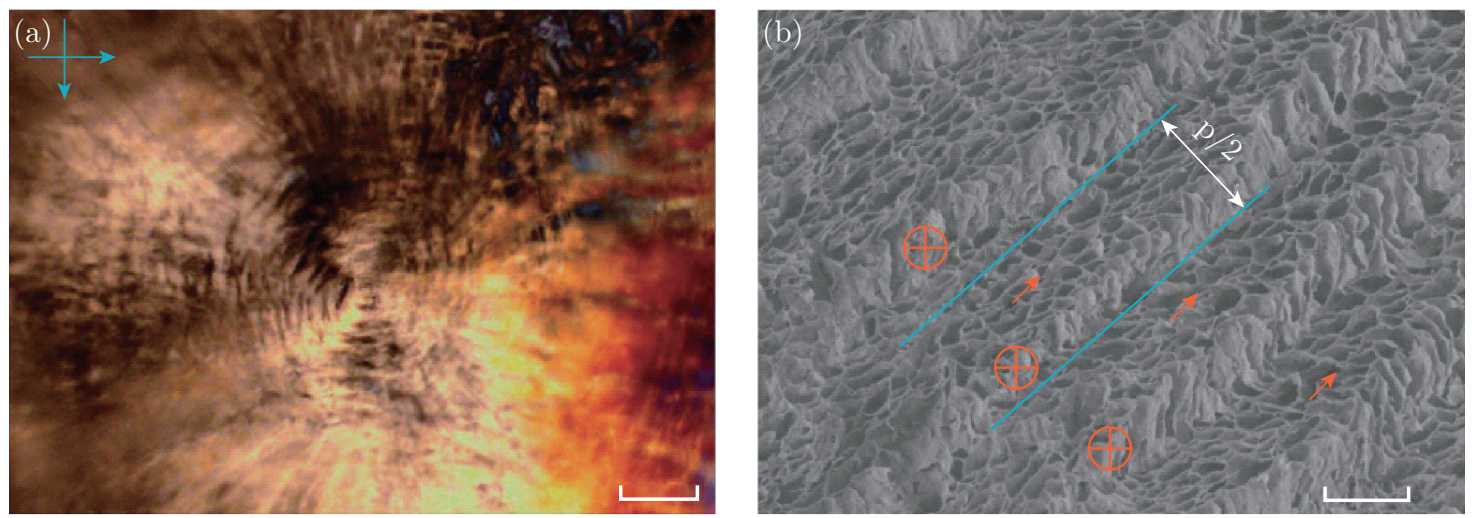

Fig. 2: (a) Polarized optical microscopic images between crossed polarizers of GO CLCs in central. (b) SEM images of morphology of LC GO. The crosses are vectors (ns) into the paper and the arrows are alone the paper. (a, b) of LC GO, scale bar $200 \mu \mathrm{m}$ [27]. Copyright 2014, Nature Publishing Group 
ratio. For graphene nanosheets especially, the most critical is to maintain a high width of graphene in solution as unique thickness of graphene. Critical size of GO sheet size of forming LC GO, with a unique thickness of graphene $0.8 \mathrm{~nm}$ and a concentration of $2.5 \mathrm{mg} \mathrm{mL}{ }^{-1}$, was established. When mean GO sheet size $\geq 1.5 \mu \mathrm{m}$, nematic phase was completely formed, i.e. LC GO was fully formed and capable to wet spinning. Mean GO sheet size is between $1.5 \mu \mathrm{m}$ and $0.7 \mu \mathrm{m}$ brought biphasic dispersions. Only discontinuous spun fibres were observed in coagulation. At mean GO sheet size $<0.7 \mu \mathrm{m}$, no nematic phase transition was found, the dispersion GO dope was totally isotropic, thus no processable.

Understanding that it is significant to have a sufficient dispersity of graphene, considering its poor interaction with most solvents, challenges remained were solved by using solvents, e.g. superacid or by chemical modifying. The former mentioned before has not been used for wet spinning yet. The latter, however, was commonly applied to form GO increasing dispersibility [21]. It was investigated that the rheological behavior of LC GO showed a non-Newtonian pseudoplastic fluid behavior. Continuous neat GO fibres combining conductive, mechanical properties were successfully spun at a minimum concentration of $0.75 \mathrm{mg} \mathrm{mL}^{-1}$. When Concentration is between $0.25 \mathrm{mg} \mathrm{mL}^{-1}$ and $0.75 \mathrm{mg} \mathrm{mL}^{-1}$ where existed a transition from biphasic to fully nematic phase, only partial discontinuous fibres were produced during wet spinning [28]. Hence the platform of spinning LC GO, containing relationship between GO sheet size, concentration and spinnability in different solvents, was established for carrying on wet spinning [20].

\subsubsection{Wet Spinning for Graphene Fibres}

First wet spun neat graphene fibre were developed in 2011 by Gao. C' group [27]. During wet spinning, LC GOs mentioned before, were injected into different coagulation baths to extract solvents and to form gel-like fibres. Gel-like GO fibres were then dried reduced into rGO fibres. As-spun fibres were slightly stretched by rotation movement in a rotating coagulation bath or drawing in a collection unit to maintain basic fibre structures during wet spinning (Fig. 3) [22].
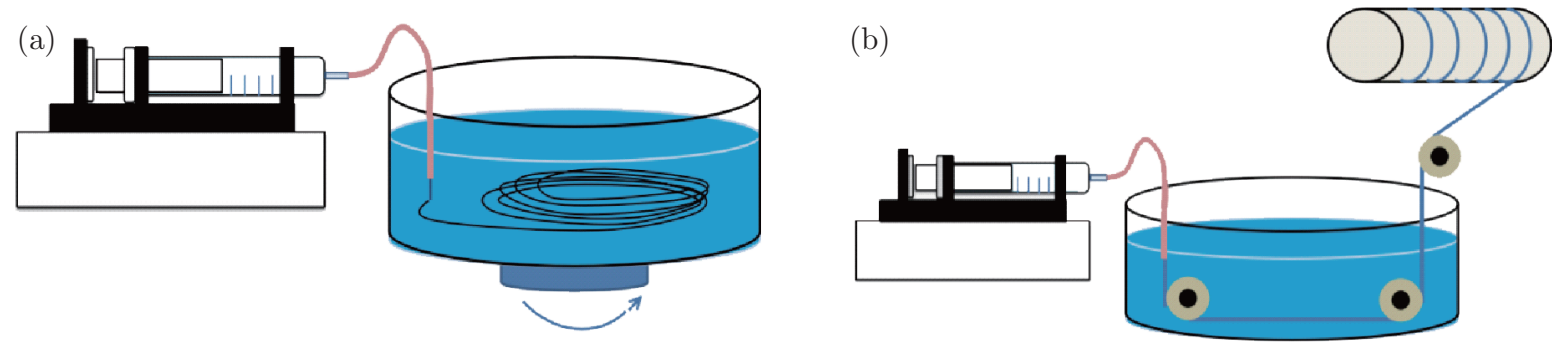

Fig. 3: Sketch of wet spinning LC GO into graphene fibres. (a) Wet spinning with a rotating collector. (b) Wet spinning with a collector unit

The formation of GO fibres was mainly based on orientation of LC GO in aqueous especially under sheer stress during uniaxial flow process. Strong attractions between GO sheets containing van der Waals and hydrogen bonds contributed to reinforce both strength along fibre axial direction and radial direction.

Coagulation bath and solvent are always considered to be key factors to wet spinning. The diffusional interchange between coagulation and dope can form solid or gel-like fibre directly, thus graphene fibre precipitations and crystalline are dramatically affected by choose of coagulation and dope. A series of coagulation bath formulations have been used as solvents, e.g. $\mathrm{KOH}$, 
$\mathrm{CuCl}_{2}$ or $\mathrm{CaCl}_{2}$ in water, ethanol or isopropanol to investigate and evaluate fibre spinnability and properties in different coagulation, seen in Fig. 4 [31]. After partly precipitation, as-spun graphene fibres were slightly drawn by a rotating coagulation or a collection unit to maintain a basic shape and further aligned to give better properties along fibre axis.
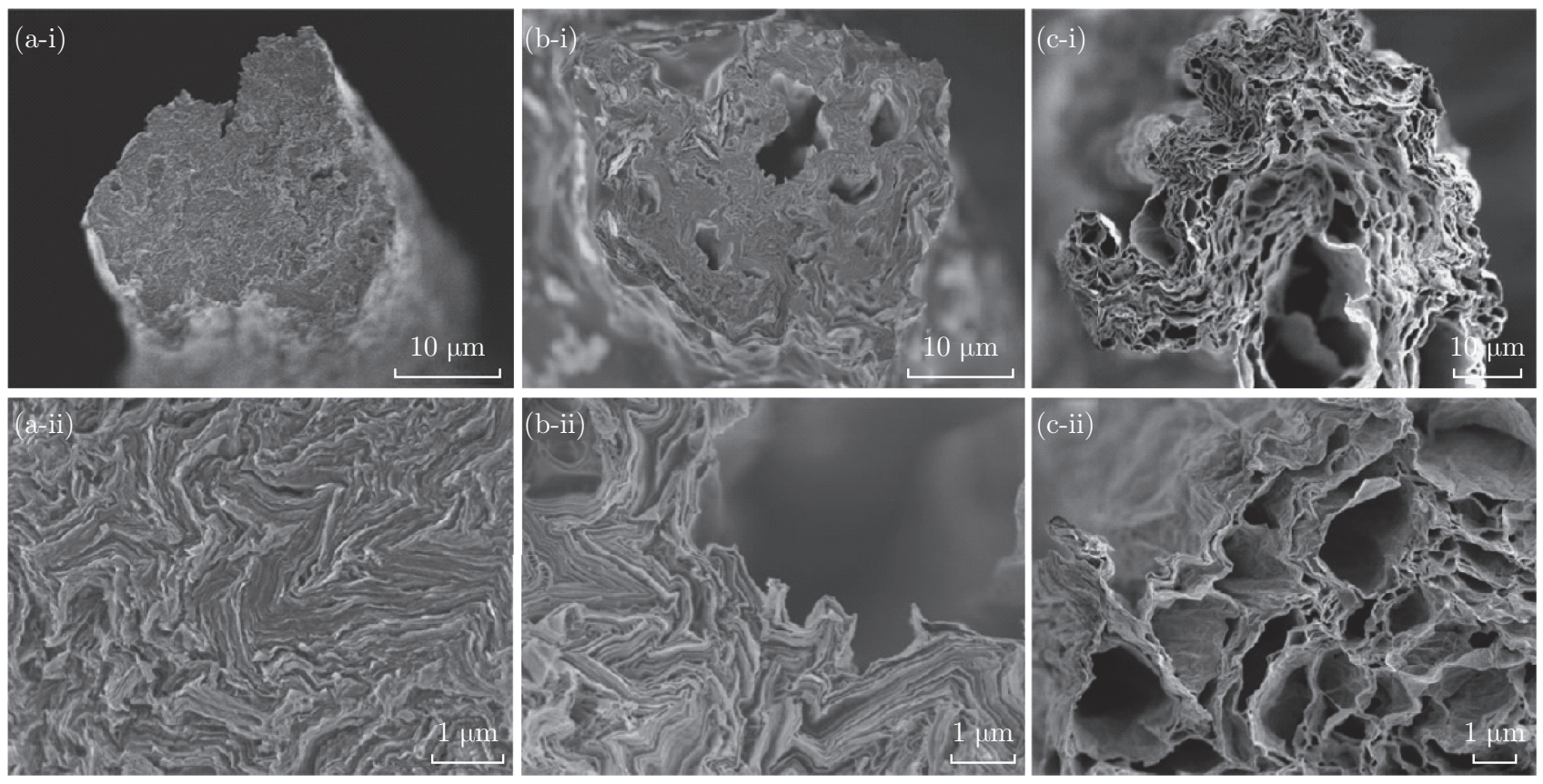

Fig. 4: The evolution of structure in different coagulation baths was compared. From (a) to (c), the architectures of graphene fibres was becoming more porous and lower dense. (a-i) (a-ii) water was used as coagulation bath leading to a slow diffusion of water from LC GO. This resulted in a quite dense structure. (b-i) (b-ii) Acetone coagulation bath resulted in the higher rate of water extraction as a result of the difference in imbibition rate, consequently leaded to higher rate of solidification and porous fibre structure. (c-i) (c-ii) In acidic condition (pH3), diffusion of LC GO dopants further fasted bringing about the difference in imbibition rate, causing much higher water extraction rate, as a consequent of which resulting in an architecture with highest porosity [31]. Copyright 2014, American Chemical Society

\subsubsection{Dry-jet Wet Spinning}

Apart from impact from alignment of LC GO, structure of GO fibres was also dominated by sheer stress formed by drawing in the spinneret and stretching during rotating or rolling. Those two factors enhancing alignment of GO sheet finally leaded to transcendental mechanical properties. Although it is greatly improved by sheer stress and gel drawing, orientation of fibres was limited by cross-linked structure in coagulation bath resulted from diffusion of $\mathrm{Ca}^{2+}$ within GO sheets. To settle this problem, a widely-used method known as dry-jet wet spinning was applied with an aim of reducing effect of cross-links. LC GO was directly drawn by air gap after jetted from spinneret following a stage of gel-forming and cross-links forming (Fig. 5).

As the first solvent, which generated LC GO, in chlorosulfonic acid as is previously stated, LC GO could be formed in a relatively high concentration of GO. Graphene oxide nanoribbons (around $4 \mu \mathrm{m}$ in length) instead of graphene nanosheets as basic fibre material successfully produced with a smooth fibre surface. Although it was reported that air gap may cause a droplet 


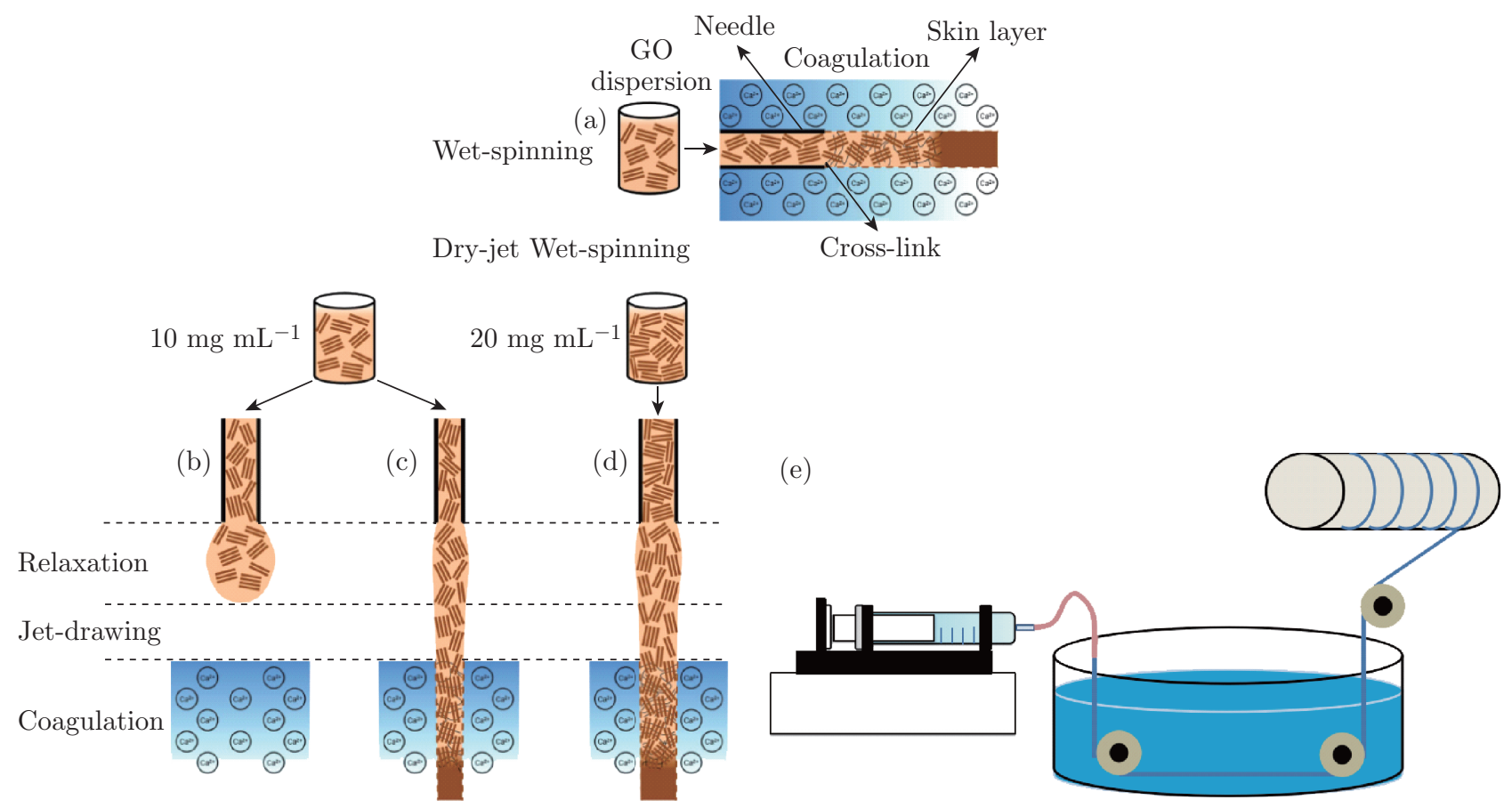

Fig. 5: Schematics of wet spinning, dry-jet wet spinning stages and process [32]. (a) During wet-spinning, a skin formed at the early stage when fibres went into coagulation bath. Cross-linking structure was then formed internally inside graphene oxide fibres. (b) Lower concentration and higher diameter form a droplet at the spinneret tip. (c) Decreased needle diameter or (d) Increased GO concentration lead to continuous dry-jet wet spinning. (c, d) fibres were drawn at the early stage and cross linked in coagulation bath. (e) Wet spinning process. Copyright 2015, Nature Publishing Group

as a consequence of which would interrupt fibre spinning, with adding graphene oxide ribbons, hundreds meters of graphene fibre with smooth surface were successfully fabricated [32, 33].

\subsection{Hydrothermal Process}

As confirmed before, dry spinning of graphene nanosheets was not a practical choice, in spite of this, hydrothermal process, seen as analogy for dry spinning, was also developed to manufacture graphene fibre. Strategy of hydrothermal process is similar to wet spinning using LC GO while replacing coagulation bath with a sealed high-temperature pipeline. This high temperature environment not only removed solvents from LC GO forming fibres, but also reduced GO into rGO. As both dramatically precipitation from solvent and reduction of GO, the shrunk fibre diameter decreased by $80 \%$ [34].

As rapidly jetted into high temperature tub, for GO dope, there is not strict limit in forming liquid crystal phase. This one-step feasible process simplified the methodology of graphene fibres. Despite showing a lack of sheer stress and tension compared with wet spinning, hydrothermal process shrunk behavior provided surface tension force and capillary force during heating reduction from gel-state to solid-state. In this case, as is reported the ratio of G-band intensity (parallel versus perpendicular) increased from 1:1 to 6:1 demonstrating that graphene nanosheets were aligned alone fibre direction [35]. $\mathrm{Fe}_{3} \mathrm{O}_{4}$ and $\mathrm{TiO}_{2}$ nanoparticles were mixed into graphene suspension and fibres to make graphene fibre composite, rendering sensitive magnetic response 
and photocurrent response respectively [34].

\subsection{Electrospinning}

However, as is highly recognised as an efficient nano technology of nanofibre process, graphene based nanofibre composites were already fabricated [35-37]. Nanofibres mat of graphene/polymer nanocomposites was electrospun applying an optical element in fibre lasers, seen in Fig. 6(a) [37]. The fibre mat rendered wideband saturable absorbance for laser pulse shaping, and attain a larger modulation depth and smaller nonsaturable loss than single-walled carbon nanotubes. Except of graphene based material made by electrospinning, GO was also used for modifying electrospun nanofibres to make fibres with versatility [36, 37]. Graphene was assembled directly onto the surface of electrospun polyamide 66 nanofibres (Fig. 6(b)). Graphene on the surface is confirmed not to form a uniform coating, but an exudative network leading to a low surface coverage. Hence, conductivity and transmittance were measured to be promoted [35].

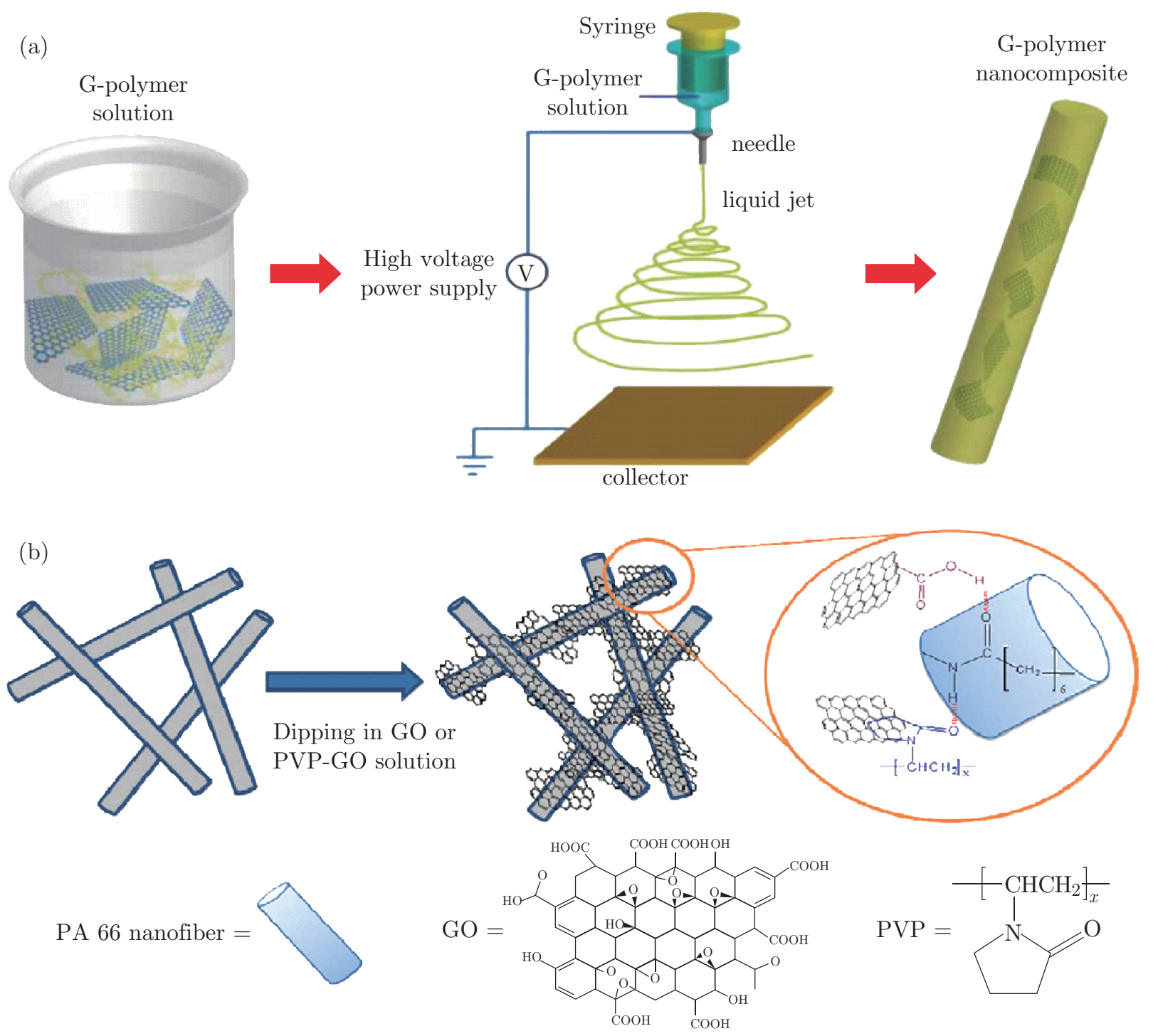

Fig. 6: (a) Schematic of the electrospun graphene/ polymer nanofibres composite. (b) Procedure of self-assembled GO or PVP-GO on the surface of PA66 nanofibers [35]. Copyright 2010, Wiley-VCH 


\section{Properties of Graphene fibres}

Making graphene fibres is actually a process of regularly aligning graphene sheets in a uniaxial direction along which the anisotropic merits of graphene are transformed into macroscopic properties. As graphene has numerous outstanding properties, it is highly expected that graphene fibres should have similar transcendental properties. Following to this aim, optimized or functional properties of graphene fibres were developed.

\subsection{Mechanical Properties}

One of the initial purpose of developing graphene fibres is to have better properties with facile methodology compared with carbon fibres. First reported graphene fibre made by a simple drawing approach does not show any records about mechanical properties. Gao's group then firstly fabricated graphene fibres prepared by traditional spinning methods with a relatively poor tensile strength $140 \mathrm{MPa}$ but decent flexibility. Although among all different methods of graphene fibre spinning, wet spinning gives best mechanical behavior, common wet spinning of neat GO or rGO fibres only gives a range of $41 \sim 182 \mathrm{MPa}$ in tensile strength and a range of $2.9 \sim 8.7 \mathrm{GPa}$ in Young's modulus, shown in Table 1. It is much lower than those of carbon fibres.

The factors dominate tensile strength are concluded as: 1) entanglement of twisted graphene

Table 1: Mechanical properties of neat graphene fibres and graphene based fibres

\begin{tabular}{|c|c|c|c|c|c|c|}
\hline Species & Ref & $\begin{array}{c}\text { Diameter } \\
(\mu \mathrm{m})\end{array}$ & $\begin{array}{c}\text { Young's } \\
\text { modulus (GPa) }\end{array}$ & $\begin{array}{c}\text { Tensile } \\
\text { strength (MPa) }\end{array}$ & $\begin{array}{c}\text { Breaking } \\
\text { strain (\%) }\end{array}$ & $\begin{array}{l}\text { Toughness } \\
\left(\mathrm{MJ} \mathrm{m}^{-3}\right)\end{array}$ \\
\hline Drawing GF & [10] & $20-50$ & & & & \\
\hline Wet spinning GOF & [27] & & 5.4 & 102 & $6.8-10.1$ & \\
\hline Wet spinning rGOF & {$[27]$} & & 7.7 & 140 & 5.8 & \\
\hline H-rGOF & [34] & 33 & & 420 & 6 & \\
\hline Wet spinning assemble GOF & [44] & 53 & 4.2 & 145 & & \\
\hline Wet spinning assemble rGOF & [44] & 43 & 8.7 & 182 & & \\
\hline rGOF/epoxy & [44] & & 6.73 & & 1.24 & \\
\hline HPM GO fibre & [31] & & 20.5 & & & \\
\hline HPM GO yarns & [31] & 120 & 29.4 & & & \\
\hline $\mathrm{GOF}$ in $\mathrm{KOH}$ & [32] & & 2.9 & 41.2 & 3.6 & 1.0 \\
\hline GOF in $\mathrm{CaCl}_{2}$ & [32] & & 5.4 & 62.9 & 6.1 & 2.9 \\
\hline Coaxial wet-spun yarn GO@CMC & [45] & & & $73-116$ & $8 \sim 10$ & \\
\hline Polymer grafted GOF & [46] & & 18.8 & 500 & & 7.8 \\
\hline GGO-HPG-GA fibres & [40] & & 20.9 & 652 & 4.1 & 14 \\
\hline $\mathrm{PCDO} / \mathrm{GBF}$ in $\mathrm{CaCl}_{2}$ & [38] & & & 842.6 & & \\
\hline U-GF & [43] & 1.6 & 282 & 1450 & 0.5 & \\
\hline Carbon fibres PAN & [21] & & $230-290$ & $3530-7000$ & $1.5-2.4$ & \\
\hline Carbon fibres Pitch & [21] & & $343-588$ & $2740-4700$ & $0.7-1.4$ & \\
\hline Graphene single sheet & {$[4]$} & & 1100 & 130000 & & \\
\hline
\end{tabular}


sheets; 2) van der Walls force between graphene oxide or graphene interlayers; 3) hydrogen bonds between formed by oxygen groups in GO sheets; 4) chemical bonds between GO or rGO sheets and other infiltrate, additive [38-40]. Continuous GO fibres showed a typical plastic deformation under tension. It is predicted to arise from displacement of GO nanosheets and stretching of folded, crumbled GO nanosheets. The failure of graphene fibres was considered to be caused by defects and pull-out process under tension. According to these factors, three strategies were taken as to be effective [22]: 1) alignment of graphene nanosheets alone fibre direction; 2) eliminating defects inside fibres; 3) inducing interlayer interaction of nanosheets to improve tensile strength.

To keep a uniform, aligned and continuous formation of GO gel-state fibre, it is essential to have a higher moving speed of collector or rotator. Rotating collector was considered as a very convenient, small-amount instrument for collection. The limitation of it is thought as not precise and no strong, reliable drawing. Collection units, however, can precisely control the drawing rate during spinning by controlling speed of collection [41].

It was also demonstrated that coagulation with $\mathrm{CaCl}_{2}$ gives better spinnability and better properties of graphene fibre [27]. The mechanism has already been discussed [28, 42], Higher $\mathrm{CaCl}_{2}$ concentration induced diffusion of $\mathrm{CaCl}_{2}$ cations, facilitated forming crosslinking between GO sheets. When LC GO goes into coagulation, GO fibre gradually precipitated, meanwhile $\mathrm{Ca}^{2+}, \mathrm{Cu}^{2+}$ from coagulation formulation goes inside GO fibres and between nanosheets forming cross-links. Mechanical properties cross-linked GO fibres were significantly enhanced. However, ultrahigh concentration, on the contrary, may cause an increasing of residual and did not show a further increasing in properties since cross-links were formed at the early stage of the diffusion.

The common main problem of graphene fibres made by different methods was lack of chemical bond of entanglement between graphene nanosheets even if they were well-aligned alone fibre, attracted by van der Waal force and hydrogen bond. One effective method was to form covalent bonds. Successively, graphene based fibres with ultrahigh tensile strength were fabricated. Gao' group combined polymers and nanoparticles into LC GO, then wet spun graphene based fibre composites were with a tensile strength of $652 \mathrm{MPa}$ were fabricated. By Bonding with $\mathrm{Ca}^{2+}$, and covalent bonding with 10,12-pentacosadiyn-1-ol (PCDO), The tensile strength of graphene based fibres was enhanced to as high as $842.6 \mathrm{MPa}$ [38]. Recently, according to Zhen Xu, a breakthrough of graphene fibres has been made to fill in the gap in tensile strength. As is reported, graphene fibres The structure of graphene sheets were restored completely with thermal annealing at 3000 ${ }^{\circ} \mathrm{C}$ to remove defects inside fibres, therefore, the highest tensile strength was reported to be 1.45 GPa [43].

Although breakthrough was already done to obtain superior properties compared with work before, there is still a huge gap in performance between ideal single graphene sheet and a single fibre. It is believed that, with offering novel approaches and optimization of spinning, graphene fibre can be dramatically improved.

\subsection{Electrical Properties}

Low-dimensional materials are of great interest to both theorists and experimentalists, owing to their novel electronic properties which arise mainly because of a host of quantum confinement effects [1]. Hence, another most important properties of graphene fibres was electrical properties, including electrical conductivity, capacitive behavior etc.

(1) Integration of chemical reduction and thermal annealing, even graphitization contribute to 
restack of graphene sheets [21];

(2) Larger GO sheets give higher conductivity of rGO fibre [32];

(3) Better alignment of GO sheets provide better [32];

(4) Incorporating may bring in superior conductivity [48, 49].

Jalili et al. fabricated neat wet spun rGO fibres (annealing at $220{ }^{\circ} \mathrm{C}$ ) of which electrical conductivity is ultrahigh up to $2508 \mathrm{~S} \mathrm{~m}^{-1}$ following a sacrificing of mechanical properties. A high specific capacitance value was detected as $409 \mathrm{~F} \mathrm{~g}^{-1}$ at a current density of $1 \mathrm{~A} \mathrm{~g}^{-1}$, much higher than best microsupercapacitors available now $\left(265 \mathrm{~F} \mathrm{~g}^{-1}\right)$ and other types of multifunctional fibers [28].

As a limitation of defects and oxygen-containing groups in neat rGO fibres, doping strategy was applied with conductive dopants (alkali metals, acids) incorporating in rGO fibres. Doping with $\mathrm{Ag}$ ions has enhanced the conductivity to $9.4 \times 10^{4} \mathrm{~S} \mathrm{~m}^{-1}$, and other may also possibly work well, as in the case of graphene transparent electrodes. Introducing Ag not only caused an improving in electrical conductivity but also enabled a high current capacity up to $7.1 \times 10^{3} \mathrm{~A} \mathrm{~cm}^{-2}$ [49]. Besides, electrical conductivity of rGO fibres were also reported to be temperature-depended, and the current carrier density of rGO fibres were $440 \mathrm{~A} \mathrm{~cm}^{-2}[22,49]$.

\subsection{Other Properties}

The thermal conductivity of ideal graphene nanosheet was ca. $5000 \mathrm{~W} \mathrm{~m}^{-1} \mathrm{~K}^{-1}$ [4]. However, the free vibration of each graphene sheet was strongly limited by increased graphene layer, i.e. thermal conductivity of multilayer graphene was much lower compared with single sheet. The thermal conductivity of rGO fibres was determined to be over $1400 \mathrm{~W} \mathrm{~m}^{-1} \mathrm{~K}^{-1}$, much higher than that of CNT fibres, 2D graphene film, implying usages in thermal management fields [50, 51].

It is reported that graphene based fibres responded to voltage, and have a magnetic property when incorporating $\mathrm{Fe}_{3} \mathrm{O}_{4}$. When mixing $\mathrm{TiO}_{2}$ into graphene fibres, a fast repeatable photocurrent response was found (Fig. 7(a)-(c) [34].

When only the upper surface of a GO fibre was reduced, the GO/rGO fibres showed reversible "fall down" and "stand up" between $\mathrm{RH}=10 \%$ and $80 \%$. Further study of the humidity-actuating performance of a twisted GO fibre demonstrated that not only did the fibre-twisting angle reversibly change, but also stretch-out and draw-back in the axial direction were observed, as shown in Fig. 7(d) [54].

\section{Application of Graphene Fibres}

Possessing the outstanding functional properties, graphene fibres were believed to have great potential in a wide range of application, e.g. functional textiles, flexible and wearable sensors, supercapacitors, electrodes of supercapacitors, energy devices, and actuators etc [51-54].

\subsection{Electrodes of Supercapacitors}

High conductivity and flexibility facilitate graphene based fibres using in electrodes of supercapacitor. Fig. 8 shows a schematic structure of the flexible supercapacitor using the CNT/G fibre 

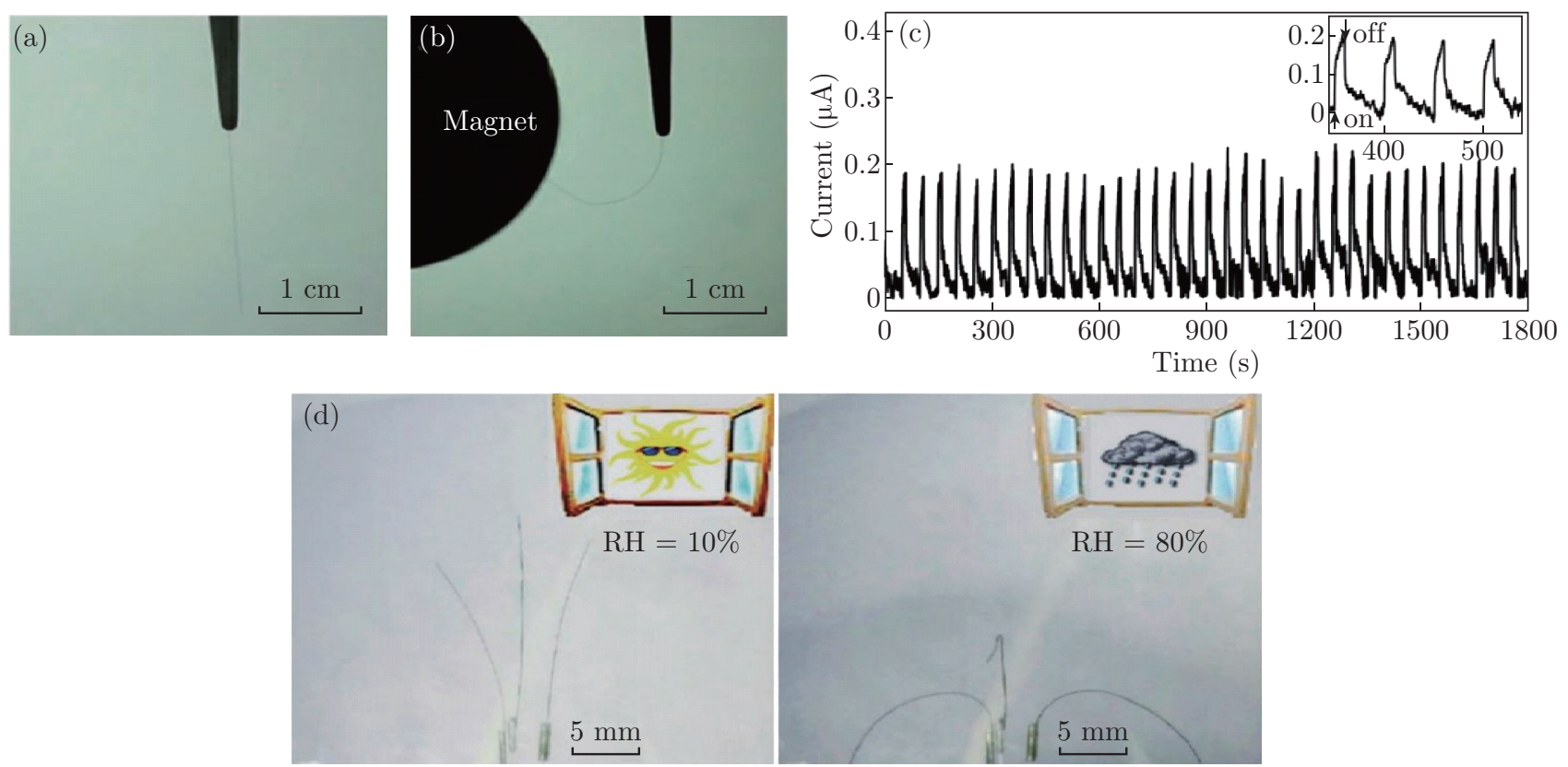

Fig. 7: Magnetic and photoelectric response of functional graphene fibres. (a), (b) the straight $\mathrm{Fe}_{3} \mathrm{O}_{4}$ /graphene fibre and the $\mathrm{Fe}_{3} \mathrm{O}_{4}$ /graphene fibre attracted to the magnet. (c) A representative photocurrent response for graphene/ $\mathrm{TiO}_{2}$ fibre upon exposure to on/off light at $20{ }^{\circ} \mathrm{C}$. The inset shows an enlarged view for a small portion of the photocurrent response curve. The sample length is $1 \mathrm{~cm}$. Copyright 2012, Wiley-VCH. (d) Responses of laser-scribed GO/rGO asymmetric fibre under varied RH $[34,54]$. Copyright 2013, Wiley-VCH

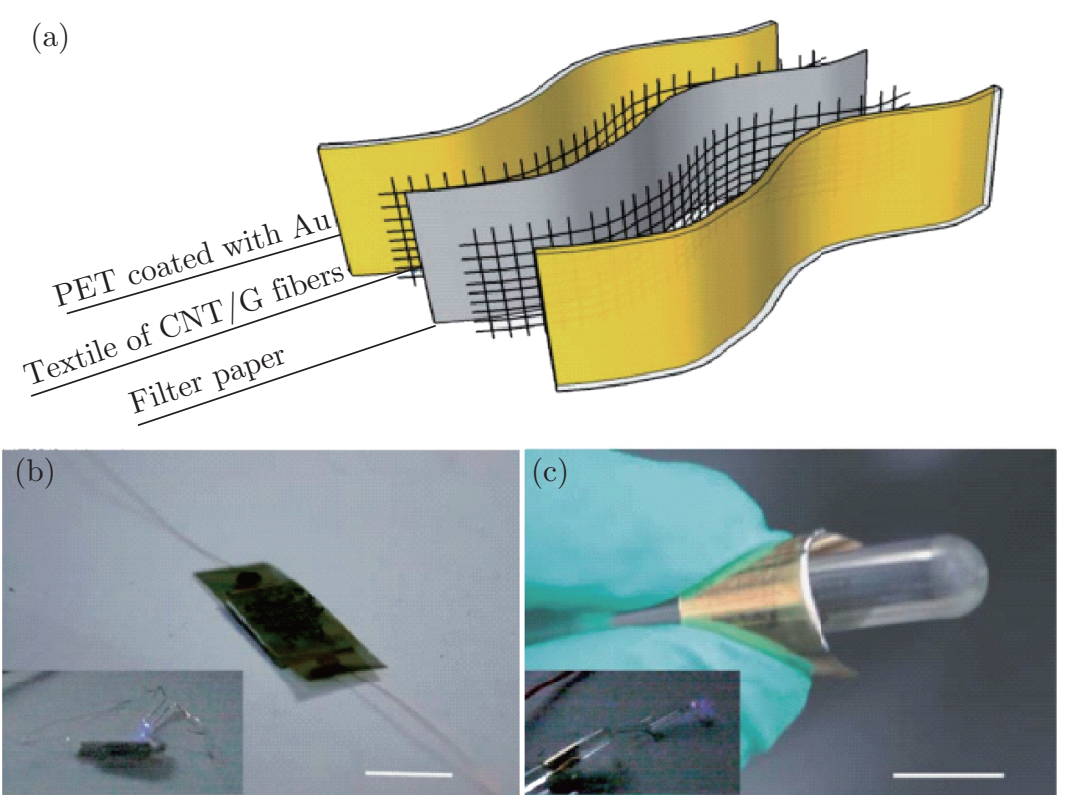

Fig. 8: (a) Schematic illustration of a multilayer textile supercapacitor using CNT/G fibres as electrodes. (b), (c) Photos of fabricated textile supercapacitor showing a light-emitting-diode (LED) lighted by this textile supercapacitor in the flat and bending states. (b), (c) Scale bar, $1 \mathrm{~cm}$ [48]. Copyright 2013, Royal Society of Chemistry

textiles as electrodes (Fig. 8(a)), and the final supercapacitors in the flat (Fig. 8(b)) and bending (Fig. 8(c)) states. A thin polyethylene terephthalate (PET) coated with Au was used as the sup- 
porting substrate and current collector. The separator is a filter paper soaked with $1 \mathrm{M} \mathrm{Na}_{2} \mathrm{SO}_{4}$ aqueous electrolyte. As shown in the insets of Fig. 8(b) and 8(c), the charged device can light a commercial LED in either flat or bending state, although the charge voltage is only $0.6 \mathrm{~V}$ [48].

\subsection{Conducting Wires}

High electrical conductivity make graphene based fibre a candidate for conducting wires. Light weighted conductive GFs are good candidates for wires in cable and other fields. Ag/graphene hybrid fibres with ultrahigh electrical conductivity showed a good resistance after 50 cycles of stretching-relaxation test and a high breakdown current density $7.1 \times 10^{7} \mathrm{~A} / \mathrm{m}^{2}$ [49]. Integrating flexibility, relatively high mechanical properties, light weight and stretchable properties, graphene based fibres were fabricated into textile (Fig. 9) [32]. Dong et al. prepared rGO/ poly (dimethylsiloxane) matrix textile [34], demonstrated hard to be deformed with stable conductivity.
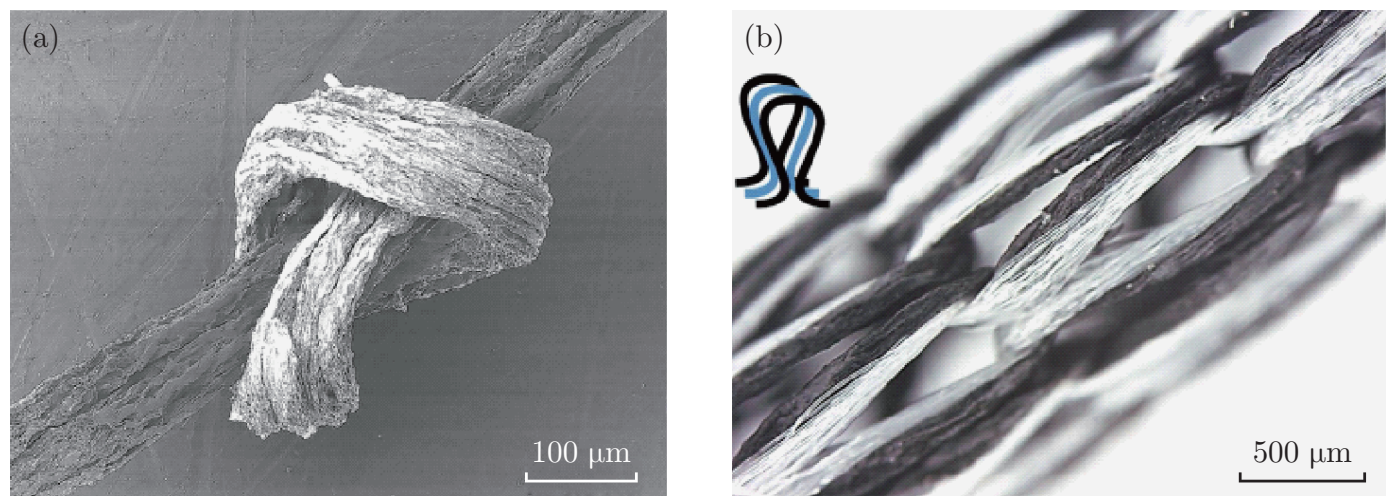

Fig. 9: (a) SEM images of a knotted yarn confirmed flexibility of graphene fibres. (b) Optical microscopy images of a textile structure co-knitted with a commercial nylon yarn using double strand of GO fibre. As shown inset, black wires represent nylon, blue wires represent graphene fibres [32]. Copyright 2015, Nature Publishing Group

\subsection{Wearable Capacitor}

Considering high surface area, capability, weavability of graphene fibres, researchers have focused on its application in wearable capacitor. Jalili et al. fabricated neat wet spun rGO fibres (annealing at $220{ }^{\circ} \mathrm{C}$ ) of which electrical conductivity is $2508 \mathrm{~S} \mathrm{~m}^{-1}$ with a sacrificing of mechanical properties [28]. After reduction, the specific surface area decreased from $2605 \mathrm{~m}^{2} \mathrm{~g}^{-1}$ to 2210 $\mathrm{m}^{2} \mathrm{~g}^{-1}$ remaining a high level. The charge storage capacity is $409 \mathrm{Fg}^{-1}$ at $1 \mathrm{Ag}^{-1}$ and rate capability is $56 \mathrm{Fg}^{-1}$ at $100 \mathrm{Ag}^{-1}$, both of which indicated a usage in supercapacitor. According to Kou et al., two coaxial graphene based fibres were twisted and then coated with insulated PVA. Combining great capacity of graphene based fibres, coaxial yarn structure, together with flexible and scalable properties, coaxial wet-spun yarn was applied in cloth supercapacitor, shown in Fig.10. This graphene based fibre cloth supercapacitor showing a capacitance of $28 \mathrm{mF}$ at the current of $10 \mathrm{~mA}$, behaved well compared with commercial supercapacitors (25 $\mathrm{mF}$ ) [45].

Apart from those applications, the temperature-dependent property in electro-conductivity gives graphene fibres potential usage in sensors. Graphene based fibres have also been illustrated to be applied in actuators, field emitters, solid-phase microextraction, springs, catalysis, etc [22]. 

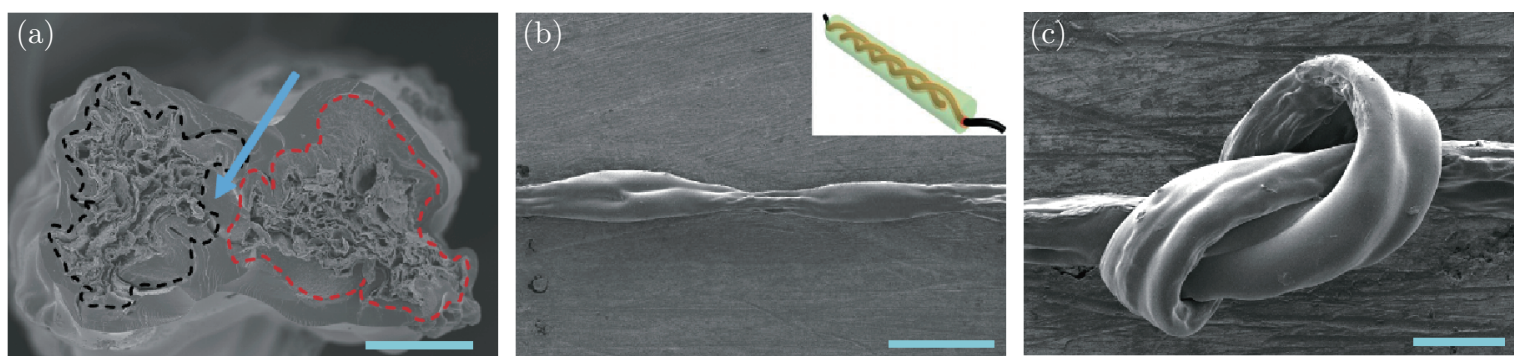

Fig. 10: SEM images of cross-section of (a) and side (b) view of a two-ply yarn. The arrow area represents a PVA $/ \mathrm{H}_{3} \mathrm{PO}_{4}$ electrolyte. Black area and red area are graphene fibres. Inset of b shows a schematic illustration of yarn. (c) SEM image confirmed that two-ply yarn can be knotted. Scale bars, $50 \mu \mathrm{m}$ (a), $500 \mu \mathrm{m}$ (b), $200 \mu \mathrm{m}$ (c) [45]. Copyright 2014, Nature Publishing Group

\section{Conclusion}

In conclusion, as is motivated by outstanding properties, not only neat graphene fibres, but also hybrid graphene based fibres have been fabricated successfully. The methodology of graphene fibres spinning includes drawing, solution spinning, thermal process, electrospinning, etc. Up to date, as showing best properties and facile steps, wet spinning liquid crystal graphene oxide is the most attractive and widely used method. The properties of wet spun graphene have been optimized by increasing size of graphene sheets, choice of solvent and coagulation bath, etc. With incorporating functional groups, ions, etc, graphene based composite fibres have shown superior and abundant properties than traditional fibres. With optimizing graphene fibres, both neat graphene fibres and hybrid graphene fibres show remarkable mechanical and electrical properties (1450 MPa and $2508 \mathrm{~S} \mathrm{~m}^{-1}$, respectively). Besides, graphene fibres also have desired actuating property, flexibility and weavability. Multifunctional properties provide graphene fibres potential in functional textiles, flexible and wearable sensors, supercapacitors, electrodes of supercapacitors, energy devices, and actuators etc. It should be pointed that despite of great progress of graphene fibres, properties of graphene fibres were still inferior to carbon fibres. Besides, conductive graphene yarns which are fabricated into wearable textile has not been developed yet. However, according to this review, it has been realized that although graphene fibres were still in its infant stage, with great efforts from scientists and researchers, great potential of both neat and hybrid graphene fibres will lead progress in technology and science.

\section{Acknowledgement}

We would like to thank the support of the EU Horizon 2020 and University of Manchester through projects with project codes 644268-ETEXWELD-H2020-MSCA-RISE-2014, AA14512 (UMRI project "Graphene-Smart Textiles E-Healthcare Network") and AA01906.

\section{References}

[1] Novoselov KS, Geim AK, Morozov SV, Jiang D, Zhang Y, Dubonos SV, Grigorieva IV, Firsov AA. Electric field effect in atomically thin carbon films. Science, 306, 2004, 666-669 
[2] Novoselov KS, Geim AK, Morozov SV, Jiang D, Katsnelson MI, Grigorieva IV, Dubonos SV, Firsov AA. Two-dimensional gas of massless Dirac fermions in graphene. Nature, 438, 2005, 197-200

[3] Novoselov KS, Jiang D, Schedin F, Booth TJ, Khotkevich VV, Morozov SV, Geim AK. Twodimensional atomic crystals. Proc Natl Acad Sci U S A, 102, 2005, 10451-10453

[4] Zhu Y, Murali S, Cai W, Li X, Suk JW, Potts JR, Ruoff RS. Graphene and graphene oxide: synthesis, properties, and applications. Adv Mater, 22, 2010, 3906-3924

[5] Kim KS, Zhao Y, Jang H, Lee SY, Kim JM, Kim KS, Ahn JH, Kim P, Choi JY, Hong BH. Largescale pattern growth of graphene films for stretchable transparent electrodes. Nature, 457, 2009, 706-710

[6] Schniepp HC, Li JL, McAllister MJ, Sai H, Herrera-Alonso M, Adamson DH, Prud'homme RK, Car R, Saville DA, Aksay IA. Functionalized single graphene sheets derived from splitting graphite oxide. Journal Of Physical Chemistry B, 110, 2006, 8535-8539

[7] Fan X, Peng W, Li Y, Li X, Wang S, Zhang G, Zhang F. Deoxygenation of exfoliated graphite oxide under alkaline conditions: a green route to graphene preparation. Advanced Materials, 20, 2008, 4490-4493

[8] Pei SF, Cheng HM. The reduction of graphene oxide. Carbon, 50, 2012, 3210-3228

[9] Cao X, Yin Z, Zhang H. Three-dimensional graphene materials: preparation, structures and application in supercapacitors. Energy Environ. Sci., 7, 2014, 1850-1865

[10] Castro Neto AH, Guinea F, Peres NMR, Novoselov KS, Geim AK. The electronic properties of graphene. Reviews of Modern Physics, 81, 2009, 109-162

[11] Wei N, Chen Y, Cai K, Zhao J, Wang HQ, Zheng JC. Thermal conductivity of graphene kirigami: Ultralow and strain robustness. Carbon, 104, 2016, 203-213

[12] Lee C, Wei XD, Kysar JW, Hone J. Measurement of the elastic properties and intrinsic strength of monolayer graphene. Science, 321, 2008, 385-388

[13] Sun H, Xu Z, Gao C. Multifunctional, ultra-flyweight, synergistically assembled carbon aerogels. Adv Mater, 25, 2013, 2554-2560

[14] Sheng KX, Xu YX, Li C, Shi GQ. High-performance self-assembled graphene hydrogels prepared by chemical reduction of graphene oxide. New Carbon Materials, 26, 2011, 9-15

[15] Xu YX, Sheng KX, Li C, Shi GQ. Self-Assembled Graphene Hydrogel via a One-Step Hydrothermal Process. Acs Nano, 4, 2010, 4324-4330

[16] Wei W, Yang SB, Zhou HX, Lieberwirth I, Feng XL, Mullen K. 3D Graphene Foams Cross-linked with Pre-encapsulated Fe3O4 Nanospheres for Enhanced Lithium Storage. Advanced Materials, 25, 2013, 2909-2914

[17] Zhao JP, Ren WC, Cheng HM. Graphene sponge for efficient and repeatable adsorption and desorption of water contaminations. Journal Of Materials Chemistry, 22, 2012, 20197-20202

[18] Alwarappan S, Erdem A, Liu C, Li CZ. Probing the Electrochemical Properties of Graphene Nanosheets for Biosensing Applications. Journal Of Physical Chemistry C, 113, 2009, 8853-8857

[19] Chen H, Muller MB, Gilmore KJ, Wallace GG, Li D. Mechanically strong, electrically conductive, and biocompatible graphene paper. Advanced Materials, 20, 2008, 3557

[20] Jalili R, Aboutalebi SH, Esrafilzadeh D, Konstantinov K, Moulton SE, Razal JM, Wallace GG. Organic Solvent-Based Graphene Oxide Liquid Crystals: A Facile Route toward the Next Generation of Self-Assembled Layer-by-Layer Multifunctional 3D Architectures. Acs Nano, 7, 2013, 3981-3990

[21] Xu Z, Gao C. Graphene fiber: a new trend in carbon fibers. Materials Today, 18, 2015, 480-492

[22] Meng FC, Lu WB, Li QW, Byun JH, Oh Y, Chou TW. Graphene-Based Fibers: A Review. Advanced Materials, 27, 2015, 5113-5131 
[23] Zakharchenko KV, Fasolino A, Los JH, Katsnelson MI. Melting of graphene: from two to one dimension. Journal Of Physics-Condensed Matter, 23, 2011

[24] Griswold PD, Cuculo JA. An experimental study of the relationship between rheological properties and spinnability in the dry spinning of cellulose acetate-acetone solutions. Journal of Applied Polymer Science, 18, 1974, 2887-2902

[25] Li XM, Zhao TS, Wang KL, Yang Y, Wei JQ, Kang FY, Wu DH, Zhu HW. Directly Drawing Self-Assembled, Porous, and Monolithic Graphene Fiber from Chemical Vapor Deposition Grown Graphene Film and Its Electrochemical Properties. Langmuir, 27, 2011, 12164-12171

[26] Behabtu N, Lomeda JR, Green MJ, Higginbotham AL, Sinitskii A, Kosynkin DV, Tsentalovich D, Parra-Vasquez ANG, Schmidt J, Kesselman E, Cohen Y, Talmon Y, Tour JM, Pasquali M. Spontaneous high-concentration dispersions and liquid crystals of graphene. Nature Nanotechnology, 5, 2010, 406-411

[27] Xu Z, Gao C. Graphene chiral liquid crystals and macroscopic assembled fibres. Nat Commun, 2, 2011,571

[28] Jalili R, Aboutalebi SH, Esrafilzadeh D, Shepherd RL, Chen J, Aminorroaya-Yamini S, Konstantinov K, Minett AI, Razal JM, Wallace GG. Scalable One-Step Wet-Spinning of Graphene Fibers and Yarns from Liquid Crystalline Dispersions of Graphene Oxide: Towards Multifunctional Textiles. Advanced Functional Materials, 23, 2013, 5345-5354

[29] Jalili R, Aboutalebi SH, Esrafilzadeh D, Konstantinov K, Razal JM, Moultona SE, Wallace GG. Formation and processability of liquid crystalline dispersions of graphene oxide. Materials Horizons, $1,2014,87-91$

[30] Khan AA, Bin-Kamarudin MA, Kidambi PR, Hofmann S, Wilkinson TD, Qasim MM. Graphene and chiral nematic liquid crystals: a focus on lasing. Rsc Advances, 5, 2015, 57437-57443

[31] Aboutalebi SH, Jalili R, Esrafilzadeh D, Salari M, Gholamvand Z, Aminorroaya Yamini S, Konstantinov K, Shepherd RL, Chen J, Moulton SE. High-performance multifunctional graphene yarns: toward wearable all-carbon energy storage textiles. ACS nano, 8, 2014, 2456-2466

[32] Seyedin S, Romano MS, Minett AI, Razal JM. Towards the Knittability of Graphene Oxide Fibres. Scientific Reports, 2015, 5

[33] Xiang CS, Behabtu N, Liu YD, Chae HG, Young CC, Genorio B, Tsentalovich DE, Zhang CG, Kosynkin DV, Lomeda JR, Hwang CC, Kumar S, Pasquali M, Tour JM. Graphene Nanoribbons as an Advanced Precursor for Making Carbon Fiber. Acs Nano, 7, 2013, 1628-1637

[34] Dong ZL, Jiang CC, Cheng HH, Zhao Y, Shi GQ, Jiang L, Qu LT. Facile Fabrication of Light, Flexible and Multifunctional Graphene Fibers. Advanced Materials, 24, 2012, 1856-1861

[35] Huang YL, Baji A, Tien HW, Yang YK, Yang SY, Ma CCM, Liu HY, Mai YW, Wang NH. Selfassembly of graphene onto electrospun polyamide 66 nanofibers as transparent conductive thin films. Nanotechnology, 2011, 22

[36] Nardecchia S, Carriazo D, Ferrer ML, Gutierrez MC, del Monte F. Three dimensional macroporous architectures and aerogels built of carbon nanotubes and/or graphene: synthesis and applications. Chemical Society Reviews, 42, 2013, 794-830

[37] Bao QL, Zhang H, Yang JX, Wang S, Tong DY, Jose R, Ramakrishna S, Lim CT, Loh KP. Graphene-Polymer Nanofiber Membrane for Ultrafast Photonics. Advanced Functional Materials, 20, 2010, 782-791

[38] Zhang YY, Li YC, Ming P, Zhang Q, Liu TX, Jiang L, Cheng QF. Ultrastrong Bioinspired Graphene-Based Fibers via Synergistic Toughening. Advanced Materials, 28, 2016, 2834-2839

[39] Kou L, Gao C. Bioinspired design and macroscopic assembly of poly(vinyl alcohol)-coated graphene into kilometers-long fibers. Nanoscale, 5, 2013, 4370-4378 
[40] Hu XZ, Xu Z, Liu Z, Gao C. Liquid crystal self-templating approach to ultrastrong and tough biomimic composites. Scientific Reports, 2013, 3

[41] Xiang CS, Young CC, Wang X, Yan Z, Hwang CC, Cerioti G, Lin J, Kono J, Pasquali M, Tour JM. Large Flake Graphene Oxide Fibers with Unconventional 100\% Knot Efficiency and Highly Aligned Small Flake Graphene Oxide Fibers. Advanced Materials, 25, 2013, 4592-4597

[42] Xu Z, Sun HY, Zhao XL, Gao C. Ultrastrong Fibers Assembled from Giant Graphene Oxide Sheets. Advanced Materials, 25, 2013, 188-193

[43] Xu Z, Liu Y, Zhao X, Peng L, Sun H, Xu Y, Ren X, Jin C, Xu P, Wang M. Ultrastiff and Strong Graphene Fibers via Full-Scale Synergetic Defect Engineering. Advanced Materials 2016

[44] Cong HP, Ren XC, Wang P, Yu SH. Wet-spinning assembly of continuous, neat, and macroscopic graphene fibers. Sci Rep, 2, 2012, 613

[45] Kou L, Huang T, Zheng B, Han Y, Zhao X, Gopalsamy K, Sun H, Gao C. Coaxial wet-spun yarn supercapacitors for high-energy density and safe wearable electronics. Nat Commun, 5, 2014, 3754

[46] Zhao X, Xu Z, Zheng B, Gao C. Macroscopic assembled, ultrastrong and $\mathrm{H}_{2} \mathrm{SO}_{4}$-resistant fibres of polymer-grafted graphene oxide. Sci Rep, 3, 2013, 3164

[47] Liu Q, Zhang M, Huang L, Li Y, Chen J, Li C, Shi G. High-Quality Graphene Ribbons Prepared from Graphene Oxide Hydrogels and Their Application for Strain Sensors. ACS nano, 9, 2015, 12320-12326

[48] Cheng HH, Dong ZL, Hu CG, Zhao Y, Hu Y, Qu LT, Chena N, Dai LM. Textile electrodes woven by carbon nanotube-graphene hybrid fibers for flexible electrochemical capacitors. Nanoscale, 5 , 2013, 3428-3434

[49] Yoon SS, Lee KE, Cha HJ, Seong DG, Um MK, Byun JH, Oh Y, Oh JH, Lee W, Lee JU. Highly Conductive Graphene/Ag Hybrid Fibers for Flexible Fiber-Type Transistors. Sci Rep, 5, 2015, 16366

[50] Balandin AA GS, Bao WZ, Calizo I, Teweldebrhan D, Miao F, Lau CN Superior thermal conductivity of single-layer graphene. Nano Letters, 8, 2008, 902-907

[51] Jalili R, Aboutalebi SH, Esrafilzadeh D, Shepherd RL, Chen J, Aminorroaya-Yamini S, Konstantinov K, Minett AI, Razal JM, Wallace GG. Wet-spinning of multifunctional graphene fibers using graphene oxide liquid crystals, 2013

[52] Cheng H, Hu Y, Zhao F, Dong Z, Wang Y, Chen N, Zhang Z, Qu L. Moisture-Activated Torsional Graphene-Fiber Motor. Advanced Materials, 26, 2014, 2909-2913

[53] Cruz-Silva R, Endo M, Terrones M. Graphene oxide films, fibers, and membranes. Nanotechnology Reviews, 2016

[54] Cheng H, Liu J, Zhao Y, Hu C, Zhang Z, Chen N, Jiang L, Qu L. Graphene Fibers with Predetermined Deformation as Moisture-Triggered Actuators and Robots. Angewandte Chemie International Edition, 52, 2013, 10482-10486 